\title{
Rethinking Graduate Recruitment Weekends: Using Slack to Make Virtual Recruitment as Effective as In-Person Visits
}

Joshua T. Gavin ${ }^{\infty}$, Allen G. Nguyen ${ }^{\infty}$, Erin E. Plasek ${ }^{\infty}$, Stephanie M. Stathopoulos, Philippe Buhlmann, Ian A. Tonks*, Courtney C. Roberts*

${ }^{\infty}$ Authors contributed equally

University of Minnesota, Department of Chemistry, 207 Pleasant St SE, Minneapolis, MN 55455

\section{ABSTRACT}

Due to the COVID-19 pandemic, the University of Minnesota shifted to a virtual graduate student recruitment weekend. Herein we report on a method to conduct a virtual recruitment weekend using the business communication tool Slack. We discuss our strategy to share both research culture and the departmental culture at the University of Minnesota with prospective students. Content creation, Slack channel curation, and a schedule of events is discussed. Additionally, comparisons to previous years acceptance rates demonstrated that the virtual recruitment weekend acceptance rates were statistically unchanged from previous years, indicating that a virtual recruitment weekend on Slack is as effective decreasing environmental impacts of graduate recruitment weekends are discussed.

\section{GRAPHICAL ABSTRACT}

\section{Allen Nguyen}

\section{\% slack}

In person recruitment weekend just got cancelled... how do you have an interactive virtual visit weekend?

$$
\text { . } 2 \text { अ }
$$

\section{Erin Plasek}

Do it on Slack! You can integrate Zoom calls for poster presentations or one-on-one meetings. Other content like pictures, Tweets, and Youtube videos can be easily shared to display campus culture and character. Prospectives can also interact with current graduate students and faculty by commenting in public channels or through direct messaging.

$$
1 \text { E } 1 \text { + }
$$

\section{Josh Gavin}

I think this would be a great opportunity to show the department remotely. And the statistics showed that it's as effective as inperson recruitment events, and even more so for students who aren't able to physically visit.

Message Erin Plasek, Josh Gavin

B B $I$ $\left\langle\right.$ \& $\frac{1}{2} \equiv: \equiv \equiv$

Aa @ (-) (1) 


\section{KEYWORDS}

Graduate education/research, interdisciplinary/multidisciplinary, internet-based learning, student/career counseling.

\section{INTRODUCTION}

Every year in February/March, the University of Minnesota Department of Chemistry welcomes approximately 100 admitted prospective graduate students to campus over two scheduled "recruitment weekends." For prospective students, these recruitment weekends are an important tool for program selection, faculty advisor selection, and more. For the department, recruitment weekends are a critical part of the fabric of departmental life and culture-an opportunity to proudly display the accomplishments of our students and the strength of our academic community. As a result of the escalating global COVID-19 pandemic, on March 9'th, 2020 the UMN Department of Chemistry was compelled to cancel our $2^{\text {nd }}$ in-person graduate recruitment weekend of 2020, scheduled for March 1315. In the subsequent 4 days, we developed and executed a comprehensive plan for an immersive "virtual recruitment weekend," and we wish to share here our experience as a successful solution to online recruitments. Outside of a pandemic situation, online recruiting provides an opportunity to reduce the cost and environmental impact of recruiting weekends and also provides a platform for increasing equity and inclusion. ${ }^{1-4}$

The move to an online recruitment weekend provided an opportunity for the department to take stock of the facets of graduate student recruitment that are (1) important in helping students make a decision in their best interest, ${ }^{5}$ and (2) valued by the department as important to positively and thoroughly convey. ${ }^{5}$ Within this framework, we identified several important aspects within two categories: "Learning, Teaching, and Research Exposure," and “Cultural Exposure," outlined below: 
- In-person meetings with prospective graduate students provide faculty with the first opportunity to present their research vision and begin the process of recruiting students to their labs (for summer research or in the Fall).

- Graduate student poster presentations provide a valuable opportunity for current students to present research progress, often for the first time outside of their respective lab groups

- Informal discussions with graduate students can provide specific details of the graduate program: how many classes do students have to take? How do students pick research advisors? What are the requirements for teaching?

Research presentations, in-person meetings, and informal discussions can expose prospective students to interdisciplinary federally-funded research centers (MRSEC, CSP, ICDC, NMGC, CSN, etc.) that span departments on campus (and universities across the country)

- In-person tours provide prospective students with first impressions on the quality of core research facilities, and individual laboratory spaces available to UMN graduate students.

\section{Cultural Exposure}

- The Department of Chemistry is committed to fostering a diverse and inclusive environment, a positive safety culture, and far-reaching scientific outreach events; this mission statement needs to be conveyed to prospective graduate students. ${ }^{6}$

- Student groups such as UMN JST (Joint Safety Team), WISE (Women in Science and Engineering), and CCGS (Community of Chemistry Graduate Students) are critical to the daily life and happiness of our current graduate students. Graduate recruiting provides these organizations with an important, high-profile forum to attract new members.

- The University of Minnesota is the only Big 10 school located in a major city. Many of our prospective graduate students are coming from places very different from the urban environment of Minneapolis and St. Paul, and graduate recruiting offers students a window into the many ways students can enjoy life in the city. 
- Finally, graduate recruiting is an important morale-boosting event for current graduate students. As one of the major annual department-wide social events, it provides students an opportunity/reward to celebrate their accomplishments and share school pride.

We recognize that our graduate students remain our most enthusiastic and effective recruiters for the graduate program. In the process of designing a recruitment weekend experience that encapsulates the nine guiding principles above, we felt it critical to make it possible for prospective students to easily, frequently, and organically interact with current graduate students in the department. Herein, we report using Slack as a unified platform for curating active/living content for a virtual graduate recruiting weekend, and compare recruiting yields across five- and ten-year departmental averages. Voluntary post-visit surveys sent out to the students who participated in the full Slack virtual recruitment weekend are discussed.

\section{Methods}

We considered several popular social media platforms for hosting the UMN virtual recruiting weekend. First, we considered direct Zoom (video chat client) links for one-on-one discussions with research faculty. However, direct video links lacked the ability for organic interactions between prospective students, current graduate students, and faculty beyond a very short discussion. Twitter was also considered, as more direct messaging and interaction between broader audiences is possible. However, given the size of our department (40 faculty, > 200 current graduate students, dozens of student groups and research centers), creating a manageable, cohesive experience through hashtags or other Twitter feed management tools seemed unwieldy. We were also concerned with privacy issues with Twitter: how can one safely share unpublished/proprietary research with prospective students in a public forum such as Twitter?

Thus, we turned to the business communication platform Slack. Slack is an internet-based platform that provides a wide range of features, including persistent chat rooms (called channels), private chat rooms, direct messaging, and file sharing.7,8 Further, various apps such as Twitter, Google Drive, and 
Zoom can be directly incorporated into Slack so students did not need to switch between different platforms to access different types of content, which we saw as critical for smooth implementation. Slack also features a powerful search tool, so content can be easily sorted and searched by appropriate tags. In fact, many research groups across the country already use Slack as a research group management tool, which we saw as an advantage for rapid adoption ( $<4$ days) by the UMN Chemistry Department for recruiting. ${ }^{7,8}$

Visitor feedback: "It [Slack] was great in terms of flexibility and ease of use!"

"I used it [Slack] for the first time, but there was no difficulty in using it."

"It felt easy to get to use Slack because everybody from UMN was using it as well."

"After a few minutes of panic it went quite smoothly. Y'all set it [Slack] up quite well, I was impressed. It also helped I'm on the east coast so I had time to check it out before it started that morning."

We have created a sample Slack workspace for readers to join and view sample content that can be accessed through https://z.umn.edu/recruitingweekendslack after approval by the corresponding authors.

\section{Slack Implementation}

Over 300 members of our department came together to create a Slack workspace to provide content that cuts across all aspects of the guiding principles outlined above. Our strategy was to provide (1) lowbarrier, real-time interactions with students; (2) real-time access to faculty; and (3) static content that could be accessed before, during, or after the recruitment weekend.

The virtual recruiting weekend was run through various "channels" (persistent chat rooms) set up within the UMN graduate recruiting Slack workspace. An example view of the workspace is shown in Figure 1. Here, students can navigate channels or directly message others by using the left sidebar (Figure 1, left). In the middle, the chat space for a selected channel is displayed (Figure 1, middle), where prospective students could ask questions or interact with current students and faculty. On the right, the "details" 
panel provides more information about a particular channel (Figure 1, right): an "about" section; a list of all members of the channel; and, importantly, a list of "pinned" items, which are permanent pieces of content that are permanently highlighted in each channel. Although the details sidebar does not show up by default, clicking on the "pin" logo (Figure 1, middle) pops it up. Chat "threads" can also be used to reply to specific posts in the main channel (Figure 2).

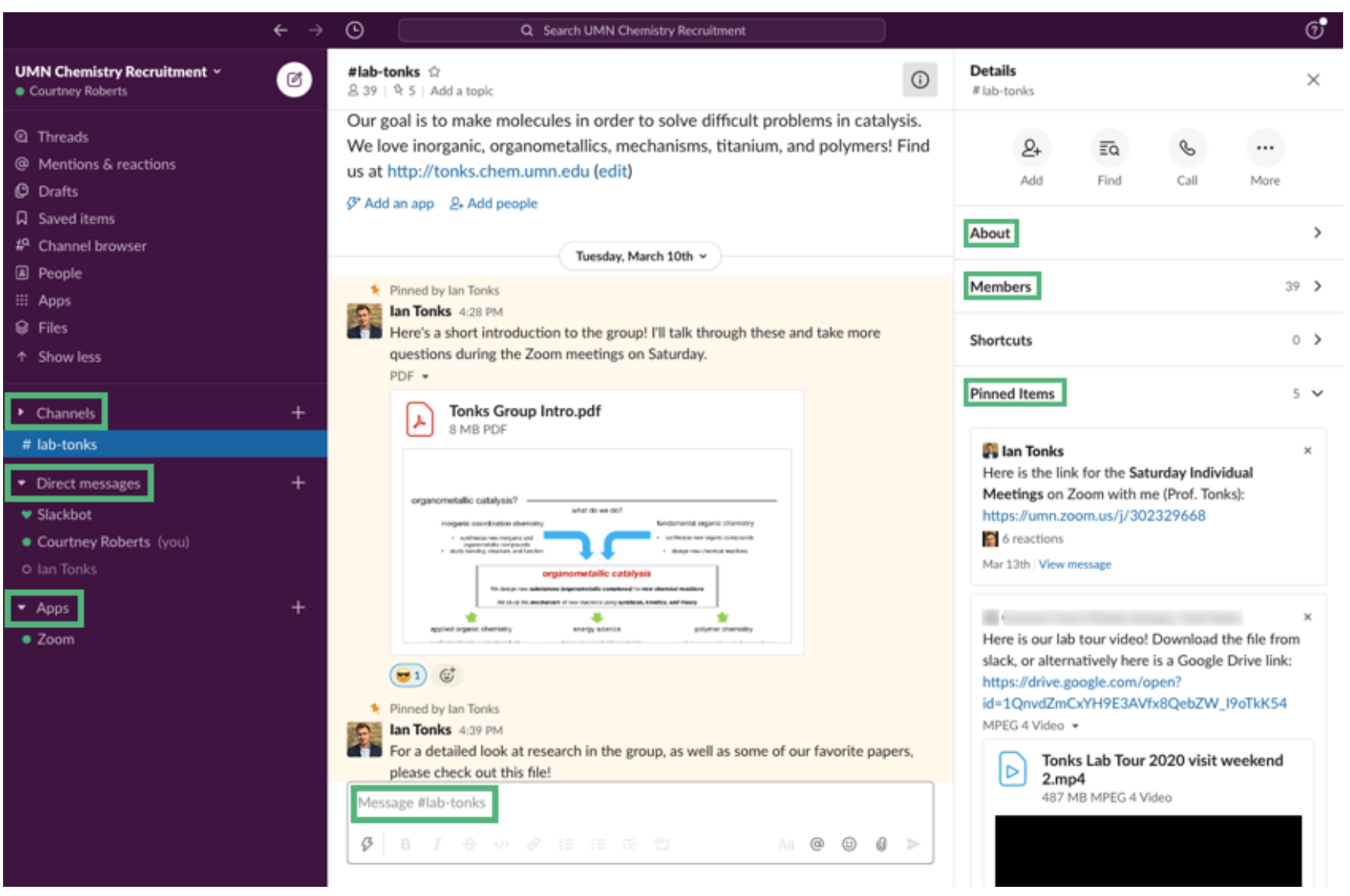

Figure 1. Example of full Slack channel for a research group 


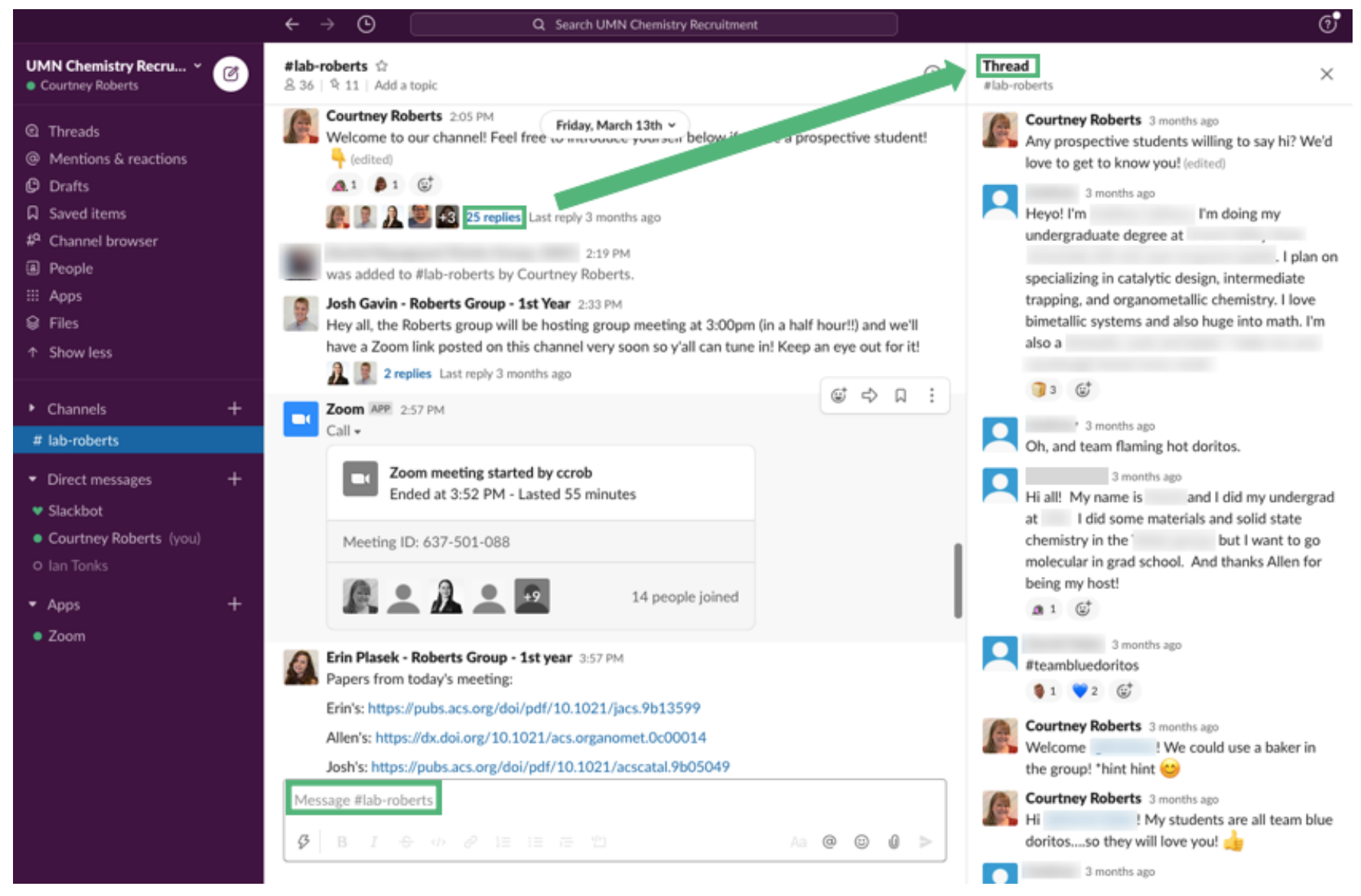

Figure 2. Example of chat threads in full Slack channel for a research group

Instructions with screenshots of the Slack interface were provided to faculty and both current and prospective graduate students (see SI for details). Current graduate students were differentiated from prospective students by listing their research group, year, and student group affiliations after their name. The prospective students were given access to the Slack workspace via email invitations $24 \mathrm{~h}$ in advance. In principle, prospective students could be invited far longer in advance than $24 \mathrm{~h}$, but given the time constraints of our rapid move to a digital platform, $24 \mathrm{~h}$ was the longest lead-time possible. Once they joined the Slack workspace, prospective students were added to relevant channels by their pre-assigned graduate student host and the coordinating administrator for the recruitment weekend, and were also given instructions about how to add themselves to other channels that may match their interests. These instructions were redundantly added to several channels to make access convenient and easy. We also invited the prospective students who had already visited on the first weekend and 
prospective international students to access the Slack workspace, although they were not the primary participants in the live events.

\section{Slack Channel Categories and Content}

Various "channels" (Figure 3) were created and divided up into categories with different purposes: (1) general channels for announcements, instructions, and help documents; (2) research groups; (3) research centers, facilities, and interest areas; (4) student groups and departmental culture exposure. Details of the content and purpose of these channels are outlined below, and a full list of channels is available in the supporting information.

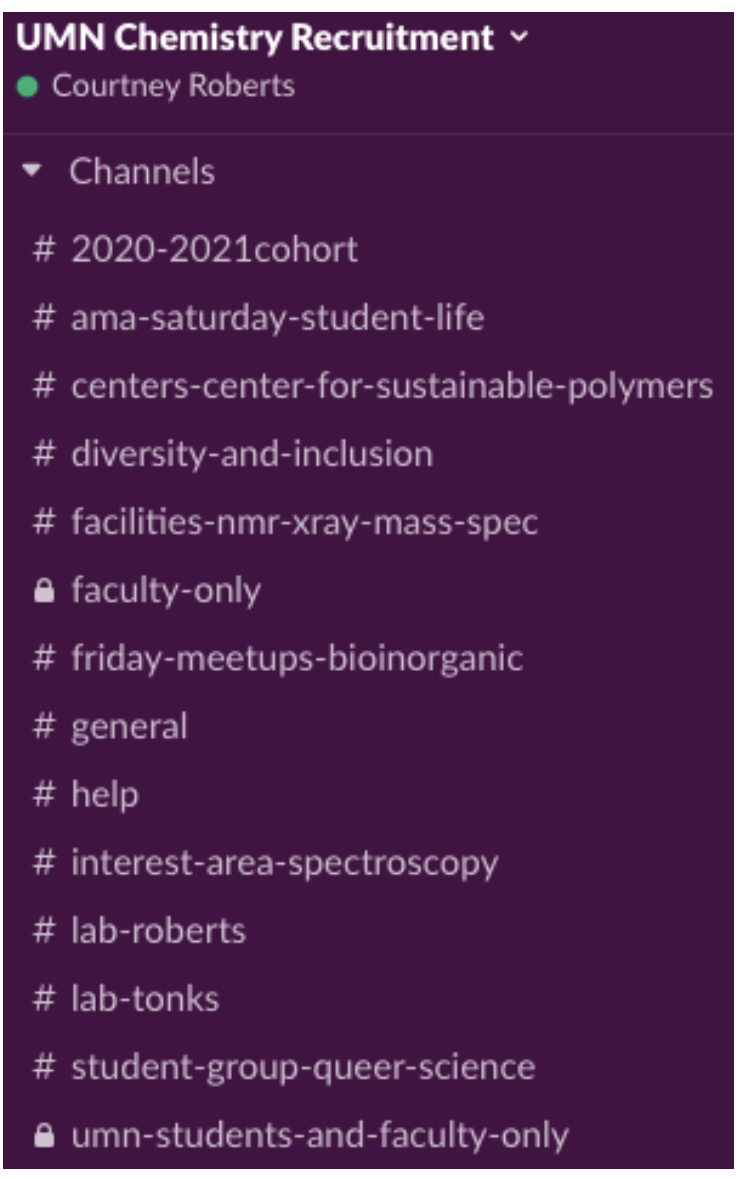

Figure 3. Representative Slack channels that can be displayed on the interface.

\section{General Channels}

\section{\#general}


Purpose: The general channel is the default landing spot of the Slack workspace, and each Slack member is added automatically. The chat in this channel was used to provide live updates and direct prospective graduate students to appropriate channels for other content (Figure 4).

\section{Persistent pinned content:}

Instructions on how to use Slack (.pdf file)

Schedule for live events for the virtual recruiting weekend (.pdf file)

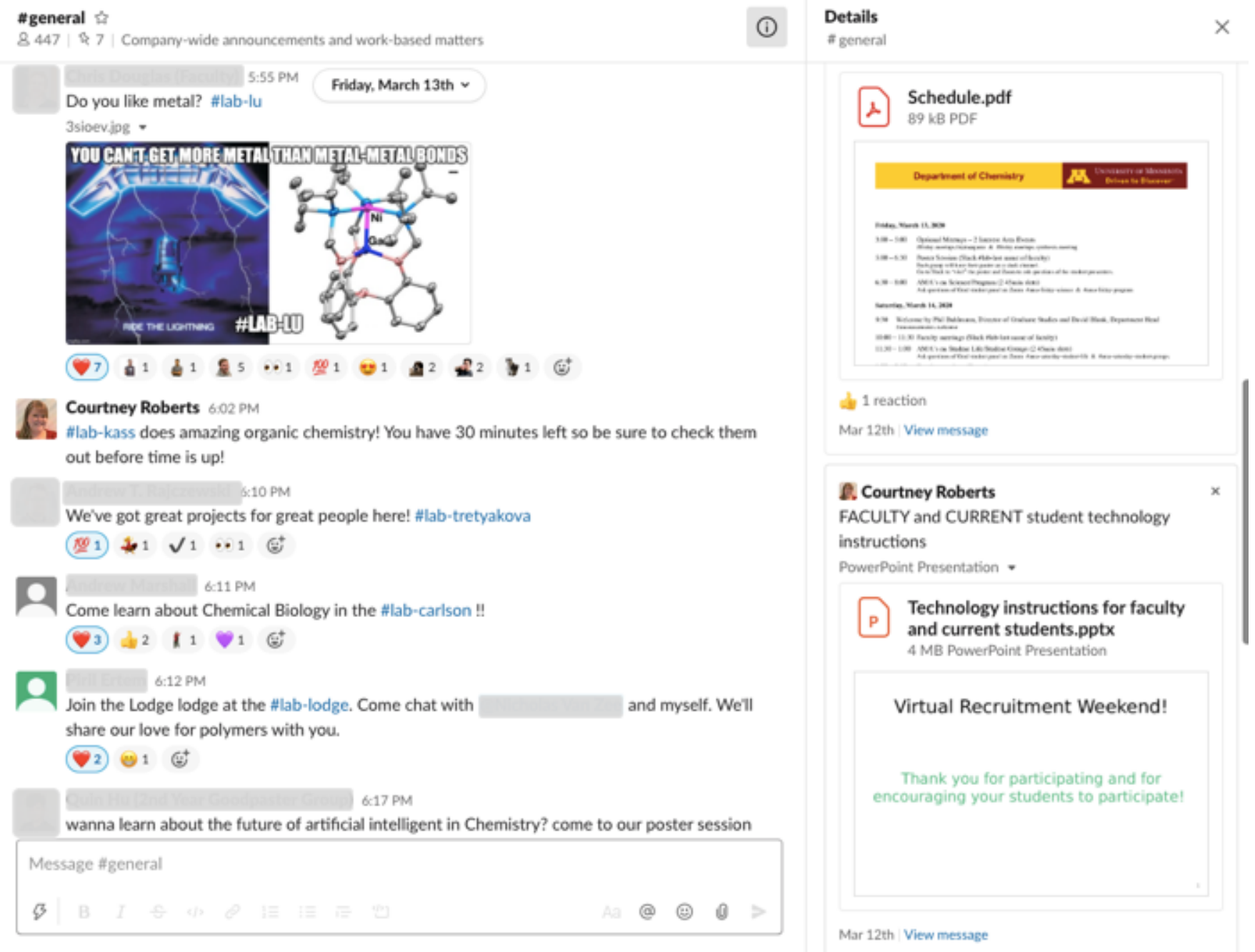

Figure 4. Advertisements and announcements from various groups and events in the \#general channel.

The retractable "Details" pane on the right side contains pinned content, including reminders for live events and links to relevant channels. 


\section{\#announcements-welcome}

Purpose: The announcement channel was the portal for important announcements, and the staging channel for the welcoming and closing events.

\section{Persistent pinned content:}

Welcome to the department video (pre-recorded): video from the Department Head and Director of Graduate Studies, aimed at showcasing campus and giving an overview of the Department of Chemistry (Figure 5). https://z.umn.edu/51rl

Zoom link for closing ceremony (live): at the end of the "live" recruiting weekend events, the faculty and students gathered for a live video send-off to highlight the collegiality of the department.

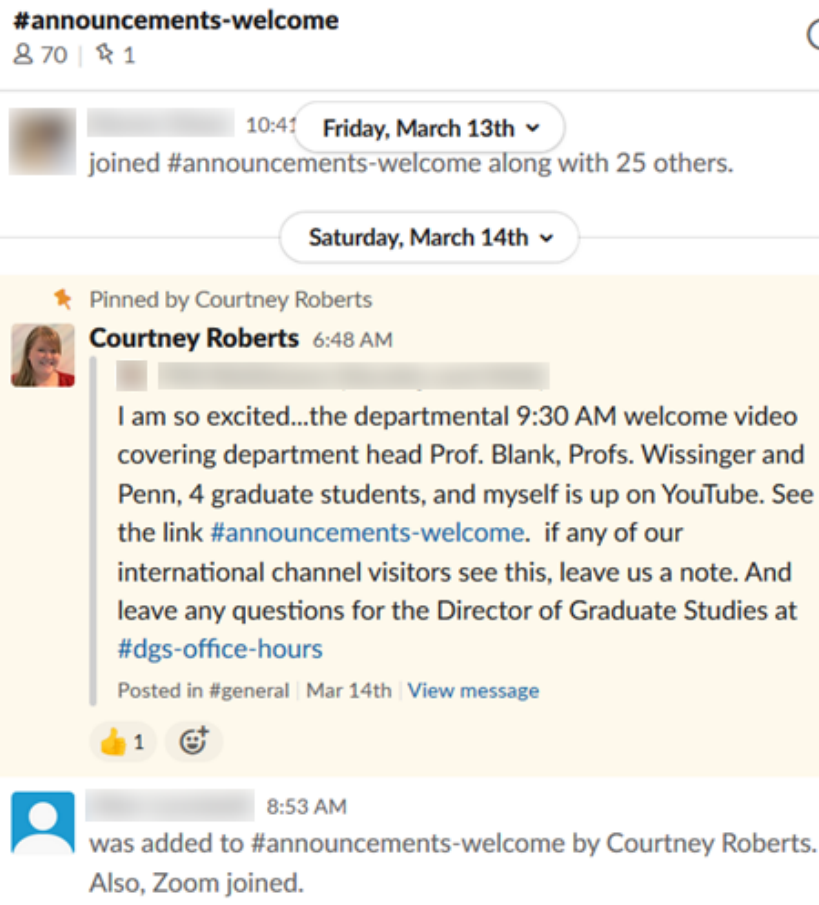

Figure 5. Examples of posts in the \#announcements-welcome channel

\section{\#student-recruitment-weekend}

Purpose: The student recruitment weekend channel served to provide general information about the UMN Department of Chemistry that students could access at any time (Figure 6). Feedback from 
students indicated that in the future, this channel and \#announcements-welcome could be combined with \#general, as there was some confusion about how these channels were differentiated.

\section{Persistent pinned content:}

$$
\text { content }
$$

about

$$
\text { the department }
$$

$$
\text { (pre-recorded): }
$$

https://z.umn.edu/5lrj,

https://z.umn.edu/51rk, https://z.umn.edu/5lrm,

https://z.umn.edu/51rl. One aspect of the recruitment weekend that was most difficult to reproduce and required the most creativity to recreate was enabling organic conversations, showcasing the departmental culture, and helping visitors to understand how they would fit in. With this goal in mind and through contributions from faculty, staff, and students, new video content was created to showcase student and faculty personalities and give visitors an idea of what it looks like to study at the university. One such video advertised to visiting students addressed common questions prospective students ask, such as about the weather, fun activities to do in the area, or typical responsibilities for teaching assistants. "Blooper reels" were also released to show the social culture of the department. These aided getting to know the department and its faculty and students.

\section{\#student-recruitment-weekend is}

$8447 \mid$ \& 4 | Add a topic

\section{Saturday, March 14th}

Courtney Roberts 7:19 AM

What are our current students and faculty waking up to in Minneapolis or St. Paul? Here is what I get to see every morning! 2 files -
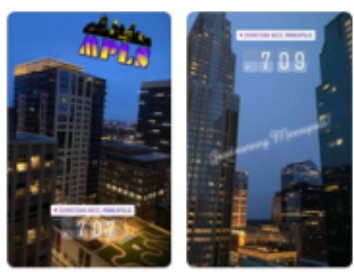

b $1: 1 \%$

\section{9:08 AM}

And here's the view out my window of downtown Saint Paul! If you look closely, you can see the train tracks in the lower right hand corner. That's the light rail station, and it's how I get to and from campus every day! If you're curious, you can ask students about it at today's \#ama-saturday-student-life session! (edited) Image from iOS - 
Figure 6. Examples of posts regarding living in Minneapolis posted in the \#student-recruitmentweekend

\section{\#help}

The help channel was set up to assist students and faculty with technology issues. It was staffed by several tech savvy graduate students and faculty volunteers for real-time troubleshooting (Figure 7).

\section{Persistent pinned content:}

Instructions on how to use Slack (.pdf file)

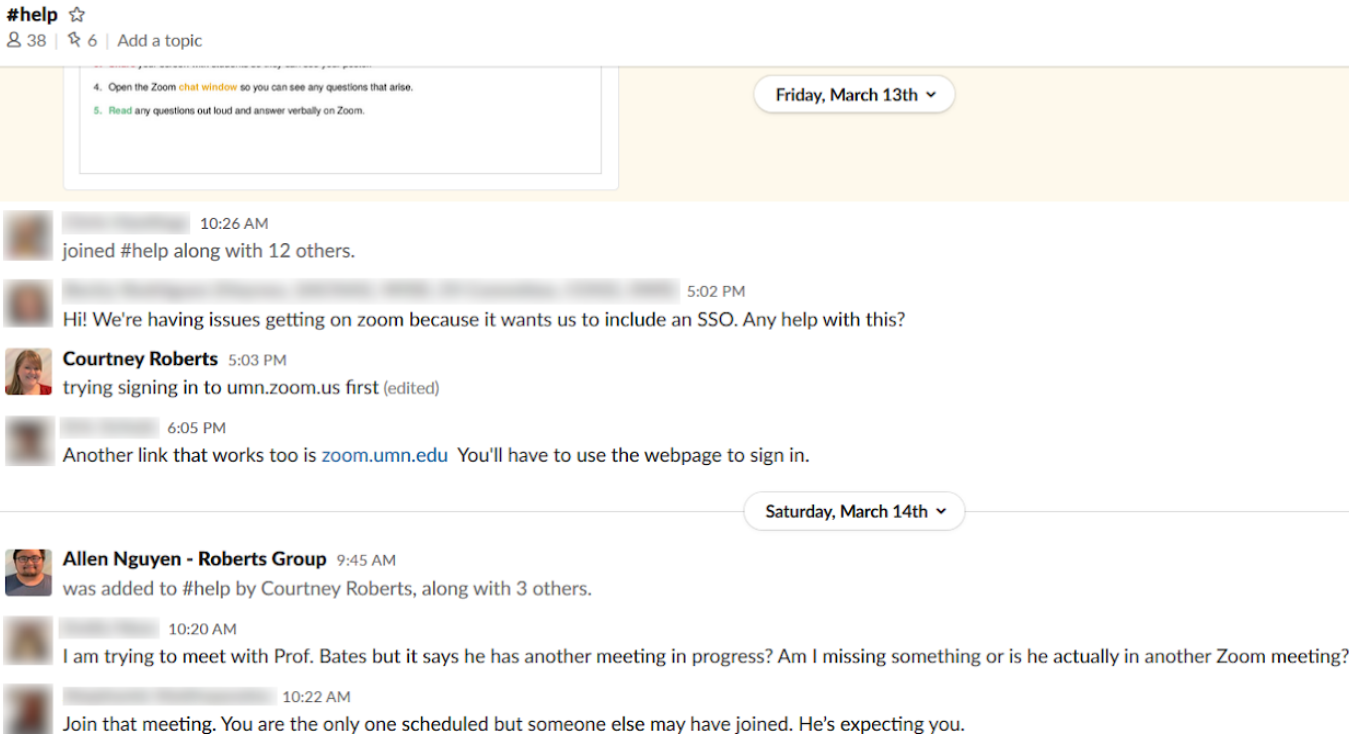

Figure 7. Examples of requests for help with technology in the \#help channel

\section{Research Group Channels}

Purpose: Research group channels served as the hub for all aspects of recruitment related to individual faculty research, and provided a forum for graduate students in a given lab to discuss science, life in the group, and more with prospective students (For example, see Figures 1 and 2).

\#lab-lastname (e.g. \#lab-roberts, \#lab-tonks)

Persistent pinned content: 
Zoom link for individual faculty meetings (live): Similar to in-person recruitment weekends, the faculty meetings were scheduled for visitors based on their expressed research interests and planned so each meeting had a minimal number of visitors to maximize direct interactions with faculty. Each faculty member had their own Zoom video conference that visitors joined at their given time, accessing the Zoom links through Slack

Research slides (.pdf or .pptx file): slides for the individual faculty meeting presentations were also provided for students to reference later.

Zoom link for graduate student poster session (live): A poster session was formally scheduled so visitors could see posters from multiple research groups during a live event. Prospective students attending the event viewed a group's poster by joining their video conference call while presenters went over their poster on Zoom using the "share screen" feature. Multiple prospective students could join at once and were able to ask questions orally or through the chat feature. Additionally, this platform allowed for some organic conversation with current graduate students. Some design features were added to the posters for the virtual recruitment, such as labelling panels with numbers to guide the audience visually while presenting.

Posters (.pdf or .pptx file): in addition to being made available during the live poster session, digital copies of the posters were uploaded to each research group channel so that students could view them at their leisure.

Lab tour videos (pre-recorded): Many research groups also decided to make their own video giving a tour of their lab space; this showed where students would spend much of their time during graduate school, giving a feel for the environment of the labs. Students were creative with their videos and gave glimpses into the group social environment as well. The tours by research groups were paired with the posters as a way of advertising for the research group as well as the school (Figure 8). 


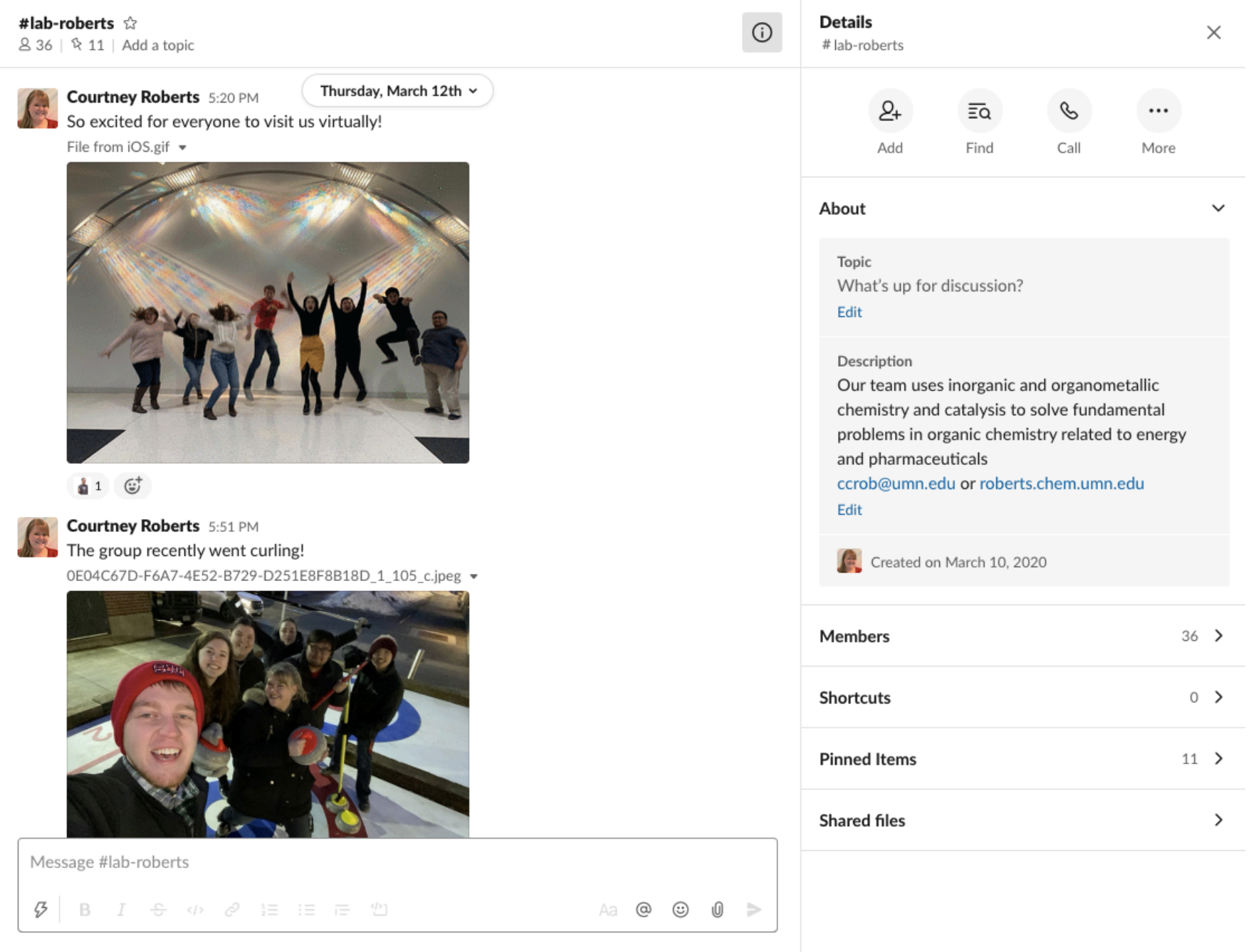

Figure 8. Example content from a research group channel that included photos from group outings

\section{Research Centers, Facilities, and Interest Areas}

Purpose: The University of Minnesota has numerous multi-group and multi-university research centers (for example NSF-CSP, DOE-ICDC, DOE-NMGC, MRSEC), cross-cutting research facilities (NMR, materials characterization, etc.), as well as strong collaborative research "interest areas" such as chemical biology, bioinorganic, and synthetic organic chemistry. Representatives from each of these entities posted static and live content to highlight the unique aspects of their respective programs. One example of each is detailed below.

\section{\#centers-inorganometallic-catalyst-design-center}


Purpose: The Inorganometallic Catalyst Design Center (ICDC) is an Energy Frontier Research Center funded by US DOE devoted to computationally-guided discovery of a new class of energy-sciencerelevant catalytic materials that are intended to be used for natural gas conversion from alkanes to alcohols.

\section{Persistent pinned content:}

ICDC welcome video (pre-recorded): featuring research highlights and faculty interviewed about the center (Figure 9)

ICDC junior investigator video (pre-recorded): interviewing junior investigators about the importance of scientific collaboration

ICDC poster (.pdf file) highlighting research being carried out by the center

Links to research groups involved in ICDC research at the University of Minnesota

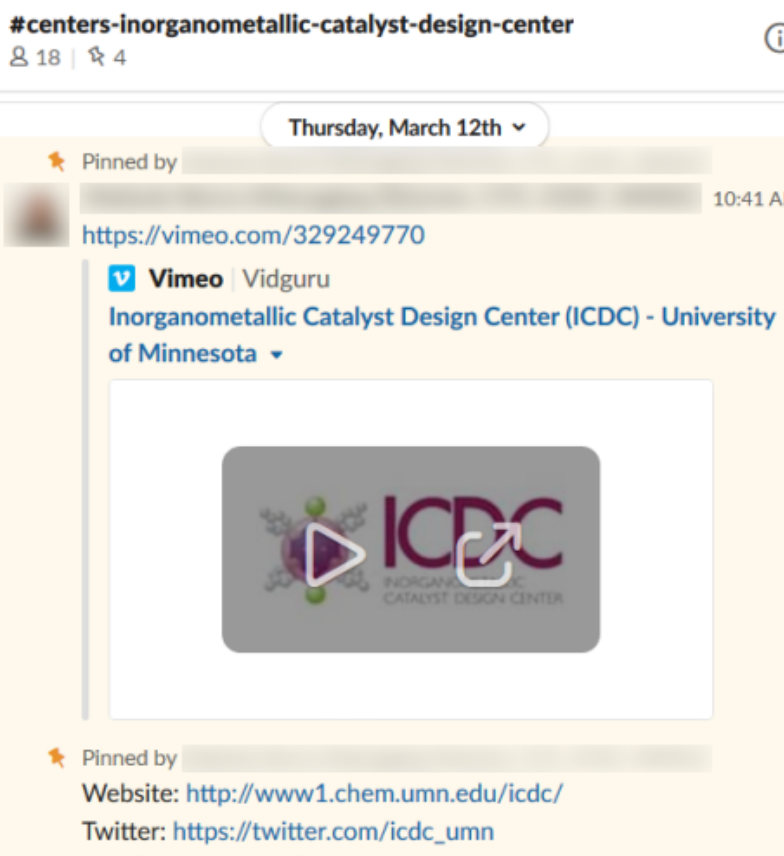

* Pinned by

Website: http://www1.chem.umn.edu/icdc/ Twitter: https://twitter.com/icdc_umn

Figure 9. Example video about a center

\#facilities-nmr-xray-mass-spec 
Purpose: This channel was created to showcase the Dow-LeClair instrument facility in the Department of Chemistry, which houses NMR, X-ray, and mass spectrometry facilities that are managed by staff scientists.

\section{Persistent pinned content:}

Facility video tour (pre-recorded): the tour featured walkthroughs of each of the instrumentation labs. This was paired with commentary on their utility to the research in the department and addresses common questions prospective students typically have (Figure 10).

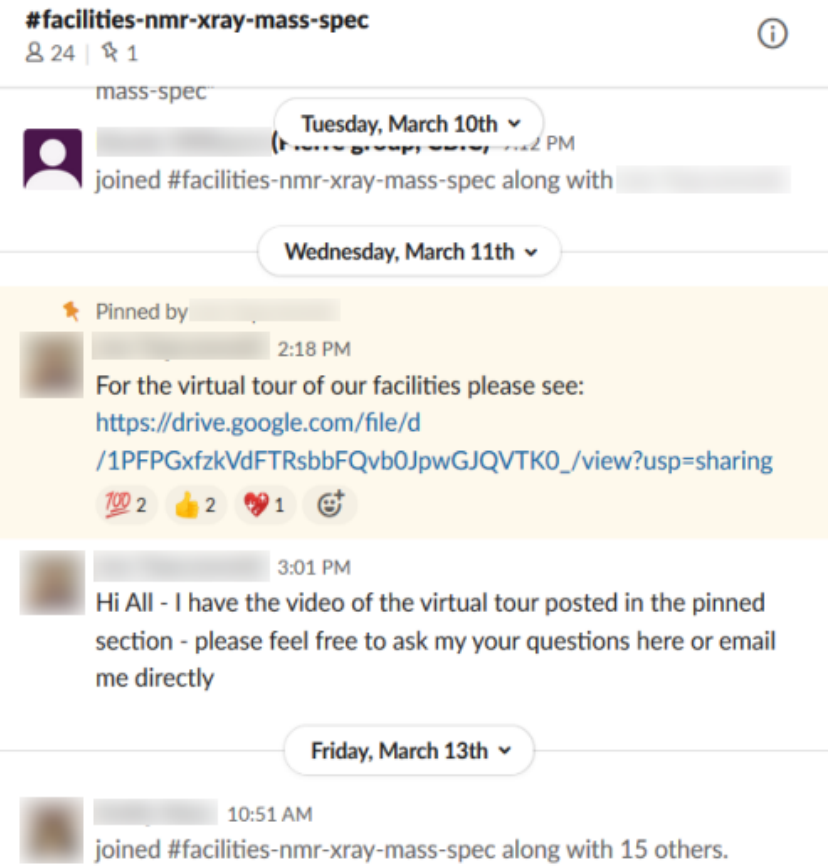

Figure 10. Example of video content posted in a facility channel

\section{\#friday-meetups-synthesis-meeting}

Purpose: Many of the synthetic organic research groups meet for a regular "synthesis lunch" meeting,

where researchers discuss research and literature. At this event, a graduate student gives an informal presentation, walking through two published organic syntheses, creating a vehicle to understand complex reactivity patterns, and building synthetic literacy. This channel was intended to allow visitors 
to participate via video conference, ask questions, and observe interactions between students and faculty (Figure 11).

\section{Persistent pinned content:}

Zoom link for synthesis lunch meeting (live): a link for the live feed of the synthesis lunch presentations by graduate students

Journal club files (.pdf): copies of the journal articles discussed as part of the live synthesis lunch journal club (Figure 12).

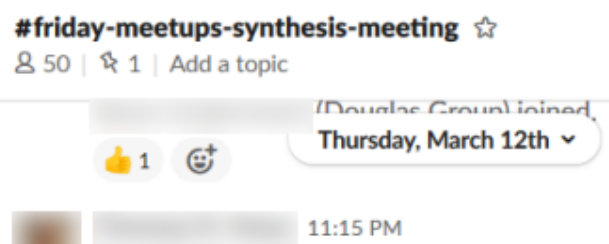
(193 K) with the usual handouts. I'll have a copy of his PDF on Zoom, that I share with the virtual folks. I'll have the computer near so that they will hear him. We can/will stop sharing to show anything the goes up on the whiteboard in 193 with the computer camera.

dis $2 \Theta^{+}$

\section{Friday, March 13th $\checkmark$}

\section{9:20 AM}

joined \#friday-meetups-synthesis-meeting along with 6 others.

$$
\text { 3:50 PM }
$$

Hi Everyone - I'm going to be joining via Zoom! See you all in a few minutes.

$$
\text { 3:53 PM }
$$

$\mathrm{Hi}$ all - sorry we could not meet in person $\mathrm{O}$ but I am happy it

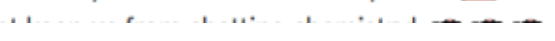

Figure 11. Example of student interactions in a meetup channel 


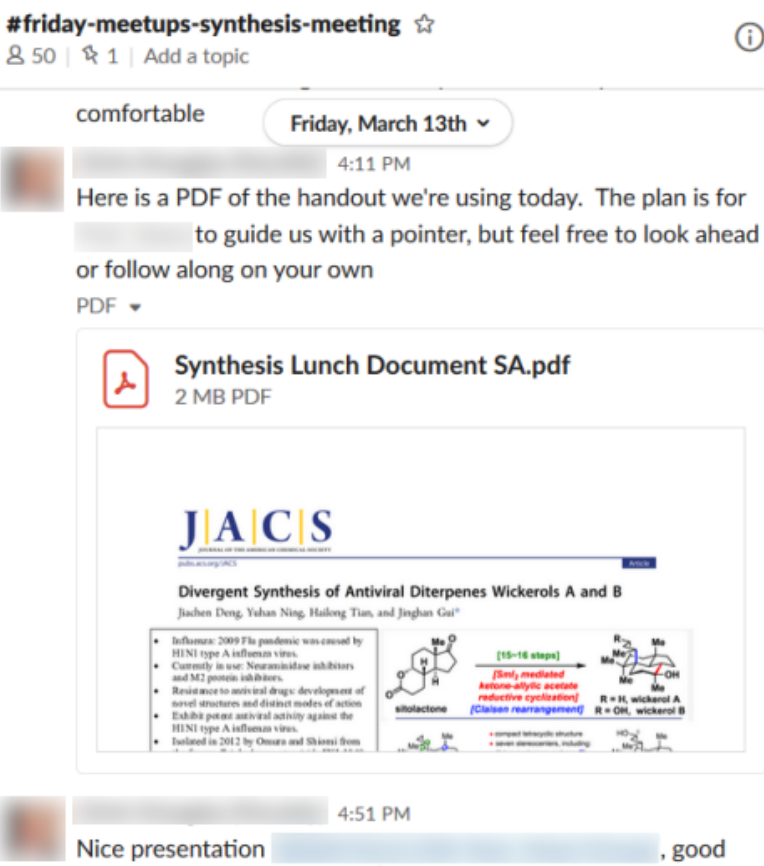

Figure 12. Example of persistent content pinned in a meetup channel

\section{Student Life, Diversity, and Inclusion}

Purpose: A large number of channels were created to represent the many department-, university- and community-based student groups that our current graduate students are a part of. An important focus of these channels was to provide real-time dialogue between current students and prospective students.

\section{\#student-group-joint-safety-team}

Purpose: The Joint Safety Team is a collaborative student group between the Chemistry and Chemical Engineering and Materials Science departments focused on cultivating safety across research labs. This channel is dedicated to raising awareness of the student group and advertising for new members (Figure 13).

\section{Persistent pinned content:}

Joint Safety Team flyer (.pdf file)

Articles published by the JST on chemical safety (.pdf files) 
Graduate student orientation presentation (.pdf file)

Pictures from disaster practice events (.jpg files)

\#student-group-joint-safety-team

$816 \mid$ \& 6

\section{Friday, March 13th}

* Pinned by

9:37 AM

You can see what the JST is up to on twitter

y twitter.com

UMN Joint Safety Team (@UMNJST) | Twitter

The latest Tweets from UMN Joint Safety Team (@UMNJST).

The Joint Safety Team (JST) is a researcher-led organization

focused on improving the culture of safety in chemical

laboratories at the University of Minnesota

* Pinned by Courtney Roberts

You can read about how the JST in the attached paper published in ACS Journal of Chemical Education in 2013

PDF -

2. Student Involvement in Improving the Culture of

Safety in Academic Laboratories.pdf

1 MB PDF

Figure 13. Example of persistent content contained in a student group channel

\section{\#diversity-inclusion}

Purpose: Diversity and inclusion is a core tenet of the mission of the University of Minnesota Chemistry Department. This channel is focused on promoting a climate that celebrates our differences and strengthens our department by embracing and working to increase our diversity (Figures 14, 15).

\section{Persistent pinned content:}

Graduate recruiting presentation (.pdf file)

Links to departmental, university, and community resources related to diversity and equity

Videos of outreach activities aimed at promoting diversity (pre-recorded) 
\#diversity-and-inclusion

$829+24$

Inadine historv.

Thursday, March 12th $~$

* Pinned by lan Tonks

lan Tonks 10:24 AM

1 Here are links to many of the student groups and resources on campus committed to enriching diversity and climate in our community:

Community of Chemistry Graduate Students:

http://ccgs.chem.umn.edu

Energy and U Outreach Program: https://chem.umn.edu/outreach /energy-and-u

Office for Equity and Diversity at UMN: https://diversity.umn.edu

Out in STEM: http://ostem.umn.edu

Society for the Advancement of Chicanos/Hispanics and Native

Americans in Science: https://gopherlink.umn.edu/organization

/SACNAS

Women in Science and Engineering: http://wise.chem.umn.edu Additional Outreach: https://chem.umn.edu/outreach/additionaloutreach

Queer Science: https://sites.google.com/site/mnqsci/

CSE Diversity Initiatives: https://cse.umn.edu/college/csediversity-initiatives

Figure 14. Example of resources pinned in the \#diversity-and-inclusion channel

\#diversity-and-inclusion

$829+24$

Friday, March 13th $~-$

Saturday, March 14th $v$

9:42 AM

$\mathrm{Hi}$ all! Just wanted to introduce myself and hopefully start a conversation going! I'm

Diversity and inclusion is very important to me and it was one of my main deciding factors when I was choosing a grad program. What I think makes UMN special is the fact that we promote inclusion just as much as diversity. Since my first semester here, the dept has made me feel like I belong! You need a support system in grad school and l've found that from the faculty, the admin staff, the teaching staff and grad students all around!

Feel free to message me with questions! (edited)

$\checkmark 4$ e)

Josh Gavin - Roberts Group - 1st Year 9:44 AM

is great, beautiful soul so absolutely take

her up on her offer to chat!!

$\checkmark 3 \uplus^{+}$

Figure 15. Example of student interactions in the \#diversity-and-inclusion channel 


\section{\#ama-saturday-student-life}

Purpose: For interactions with current graduate students, AMA (Ask Me Anything) panels were set up to address different topics about the university such as the graduate program, student life in the metro area, or student groups the recruits may be interested in participating with. Each panel had a range of current students as panelists to span a wide reach of experiences (Figure 16).

\section{Persistent pinned content:}

Zook link AMA panel (live)

\section{\#ama-saturday-student-life}

864 ? 1
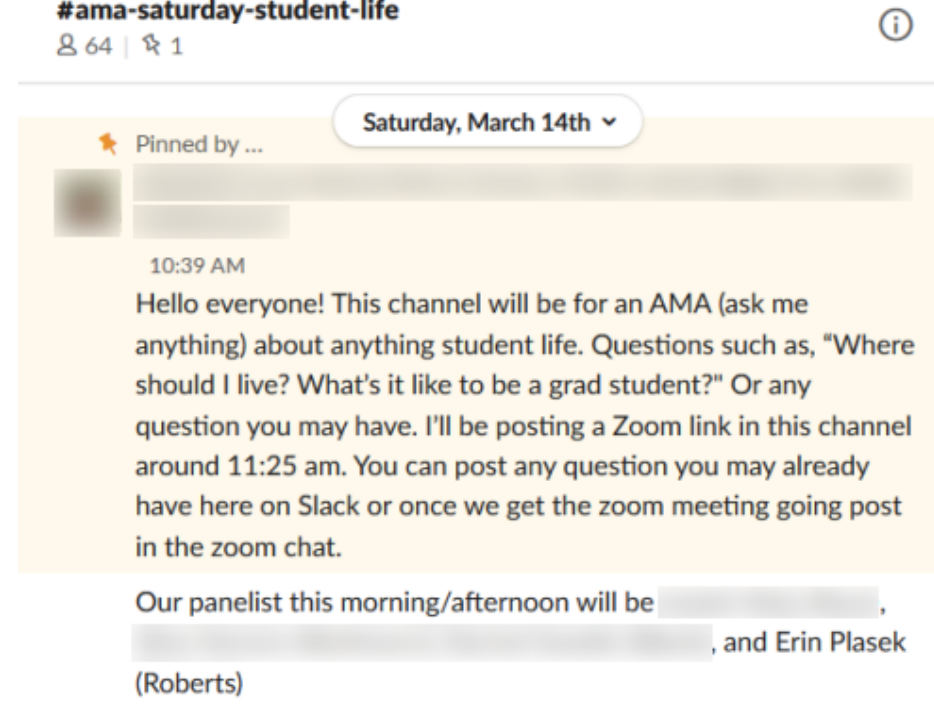

Tuesday, March 24th

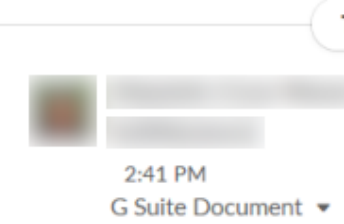

Figure 16. Example of content in an AMA channel

\section{Implementation: Visit Weekend Schedule}

In order to mimic an in-person recruitment weekend that visitors may have already experienced at other

universities and convey the same information given in a personal visit, the same schedule structure was preserved. Additionally, students received schedules with the names of the Slack channels where events and content were hosted (Figure 17). In order to not overwhelm students with virtual meetings, several 
styles of events were created: those with 1) static content, i.e., facilities tours and the welcome video, 2) live events where students could passively participate or chose their level of participation, e.g., AMA panels and meetups, and 3) live events where students were actively engaged, i.e., the poster session and faculty meetings. Graduate student hosts--who are a regular feature of UMN graduate recruiting-were enlisted to help their assigned prospective students with Slack and Zoom by offering to test-drive the platforms prior to the official start of the schedule. It should also be noted that when asked the question "Which events should be eliminated?" in the post-visit survey, none of the students responded that any of the events should be eliminated in the future.

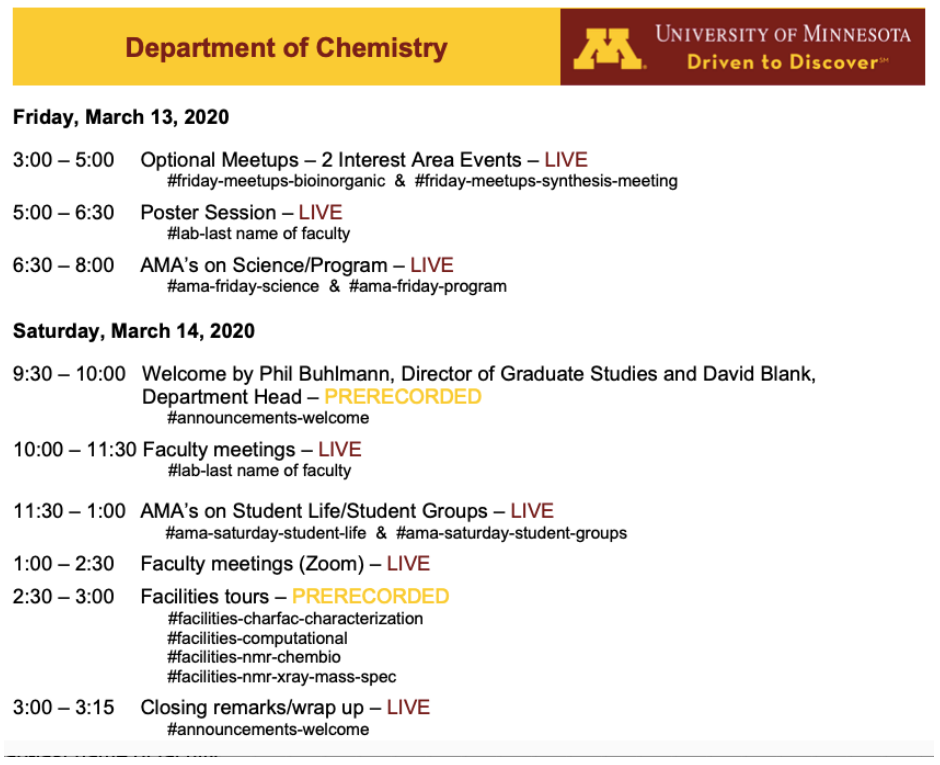

Figure 17. Schedule that virtual visitors received that included channel names and whether events could be accessed live or were prerecorded

\section{Optional meetups Friday 3:00-5:00 pm}

As with in-person visits, the Department of Chemistry at UMN hosts a number of informal "meetups" that students who arrive before the official recruitment weekend start can attend. These events began before the others scheduled for recruitment, introducing the department in a relaxed atmosphere without active involvement from visitors being necessary. We hosted these virtually and invited students 
to attend via a Zoom link provided in the appropriate Slack channel (\#friday-meetups-bioinorganic, \#friday-meetups-synthesis-meeting).

Visitor feedback: "I enjoyed all of the events. The "lunch meetings" on Friday were a nice touch to see students and faculty interact in a normal grad school environment.”

"I really enjoyed getting to sit in on the bioinorganic meeting Friday afternoon."

\section{Poster Session Friday 5:00-6:30 pm}

A poster session was formally scheduled so visitors could see posters from multiple research groups at the same event, much like they would at an in-person poster session. Recruits attending the event viewed a group's poster by joining a video conference call through the Zoom link provided in the appropriate channel (e.g., \#lab-roberts). Graduate student presenters went over their group's poster on Zoom using the "share screen" feature. Multiple recruits could join at once and were able to ask questions orally or through the chat feature, and could come and go as they pleased, switching between group zoom meetings at their leisure. We recommended that students attend posters between 5-20 minutes. Additionally, the poster sessions bled into continued organic conversations with current graduate students in the chat section of the respective lab channels.

Visitor feedback:

"Poster session was run very well, as were the group meetings."

"Useful - the student poster sessions (because of the opportunity to talk to students and to learn about specific research)...”

\section{AMA Panels Friday 6:30-8:00 and Saturday 11:30-1:00 pm}

With the goal of showcasing the culture of the department, we heavily focused on allowing prospective students to organically interact with current graduate students through concurrent AMA panels on both Friday and Saturday. Because visitors were not able to ask questions in person, the AMA panel provided a platform to encourage questions and answer them in one place so all prospective students benefited. The two Friday student panels were oriented toward aspects related to scientific questions and questions 
about the graduate program, while the Saturday student panels were more oriented toward student life and culture in the department (Figures 18 and 19). Students were encouraged to switch between panels as their questions got answered.

Visitor feedback: "AMA programs were very useful!"

"AMAs were also helpful, and it was nice that they were more-or-less separated by category."

"The grad-student life talk was very useful, especially being able to ask questions via chat."

"I also really appreciated the student panels."

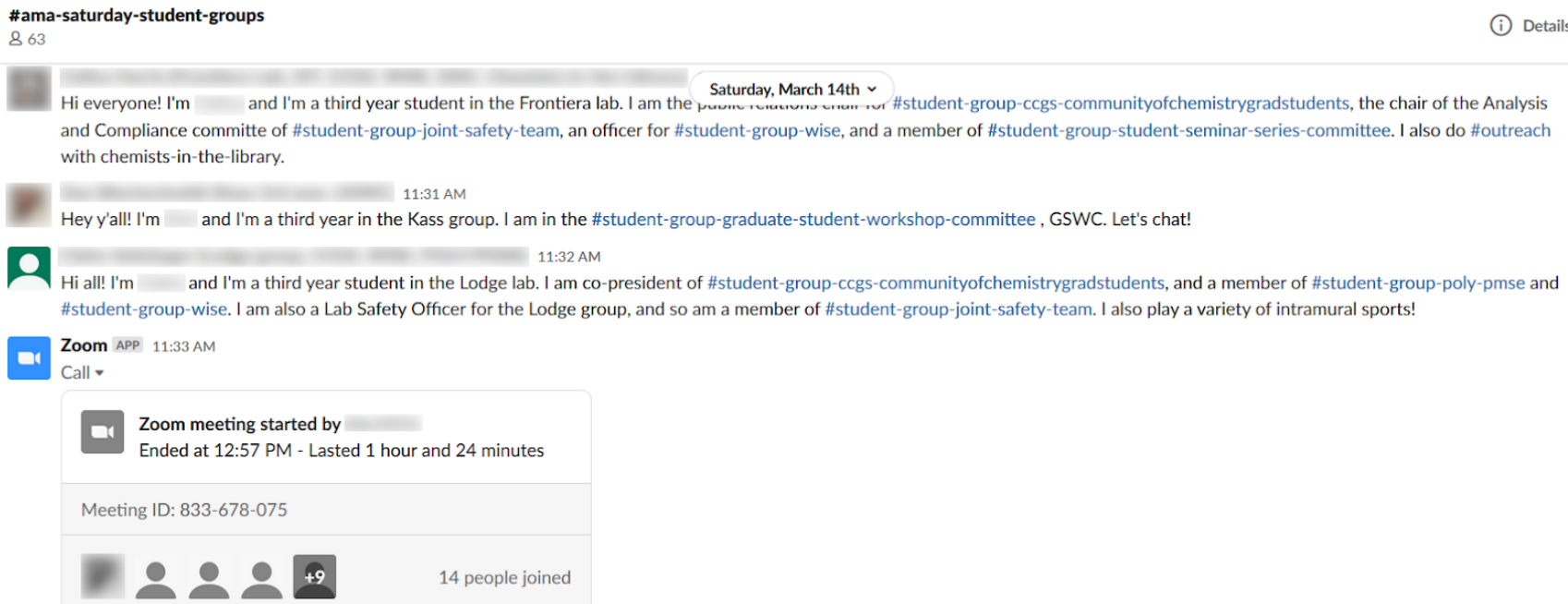

Figure 18. Graduate students introduced themselves on their channel before a live Zoom "ask me anything" panel about student groups. The students tagged the groups and activities they are involved with so that perspective students could easily find more information. 


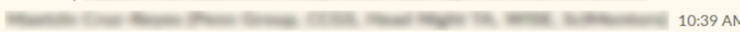

Hello everyone! This channel will be for an AMA (ask me anything) about anything student life. Questions such as, "Where should I live? What's it like to be a grad student?" Or any question you may have. I'll be posting a Zoom link in this channel around 11:25 am. You can post any question you may already have here on Slack or once we get the zoom meeting going post in the zoom chat.

Our panelist this morning/afternoon will be

\section{Tuesday, March 24th}

GSuite Document *

(1) Student Life

12. Document from Google Drive

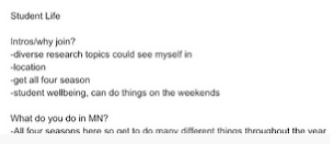

Figure 19. An example of an "ask me anything" channel. A Zoom session link was posted and pinned to the page. A record of the panel responses was taken and posted on the slack channel following the Zoom session.

\section{Welcome to the Department Video - Prerecorded but suggested for viewing Saturday 9:30-10:00} am.

During recruitment weekend, a wide variety of information about the program and departmental culture is disseminated at a kick-off presentation on Saturday morning. In order to capture this content, a video from our Director of Graduate Studies and Department Head was curated. The students were encouraged to watch this prerecorded video at the start of the day but it remained persistent content for those in time zones where it was too early in the morning to watch. Recognizing that prospective students would not be able to walk across campus and get a feel for its size and its many facilities, parts of this video were filmed in the chemistry stockroom, several research facilities across campus, the recreation center, and in front of one of the light rail stations, to name just a few locations.

Faculty Meetings 10:00-11:30 am and 1:00-2:30 pm

Using the pinned content and Zoom links in each group's channel, faculty meetings were organized into 30 minutes slots. Most faculty members presented slides via screen share or gave a verbal overview of 
their group's research and culture. Faculty members also left time at the end of each meeting to answer questions.

Visitor feedback "Meetings with professors were the most useful."

"Useful - ...faculty chats (because of the opportunity to meet faculty members and learn about groups)..."

\section{Facilities Tours 2:30-3:00 pm}

Time was built into the schedule so that students could view the pre-recorded video content and other static content on the facilities Slack channels. Student volunteers and TAs were also available during this time to answer visitor questions in the relevant Slack channels.

Visitor feedback: "The facilities tours I did see were nice overviews."

"I'm not sure anything should be eliminated. I found the tours less useful but I think that was the online method, and that at an in-person event, they make sense."

\section{Closing Remarks 3:00-3:15 pm}

In order to solidify the impressions of our collaborative, adaptable, and progressive departmental culture, we had a live event to close out the weekend. Current graduate students and faculty gathered in a large lecture hall (with social distancing) to enjoy an ice cream social. Our Department Head, Director of Graduate Studies, and organizers all gave wrap up remarks about the virtual event that were broadcast live to students who tuned in. We wanted to leave visiting students with a final impression of a room full of UMN chemists who had come together over six days to make the best out of a challenging situation that profoundly impacted our society.

\section{Outcomes}

In order to measure the effectiveness of a virtual recruitment weekend, we compared the success of our virtual recruitment weekend with the previous year, five-year average, and ten-year average (see below), measured as a function of acceptance rate of offers extended. To examine the data, the prospective student population was broken down into categories: (1) students who attended the first in-person 
recruitment weekend of 2020 (first weekend visitors); (2) students originally scheduled to attend the second in-person recruitment weekend that were instead invited to the virtual recruitment weekend (second weekend visitors); (3) students who did not plan on attending either of the in-person recruitment weekends, including international students as well as domestic students who did not register for either recruitment weekend (non-visitors).

\section{Overall acceptance rate}

The overall offered student acceptance rate in 2020 (36.3\%) is higher than that of the previous year (29.7\%), the average of the previous five years (32.5\%), and the average of the previous ten years $(32.2 \%)$. The increased acceptance rate was not statistically different from any of the compared rates, with pvalues of 0.188 (2019), 0.225 (five year average), and 0.296 (ten year average) respectively (Figure 20).

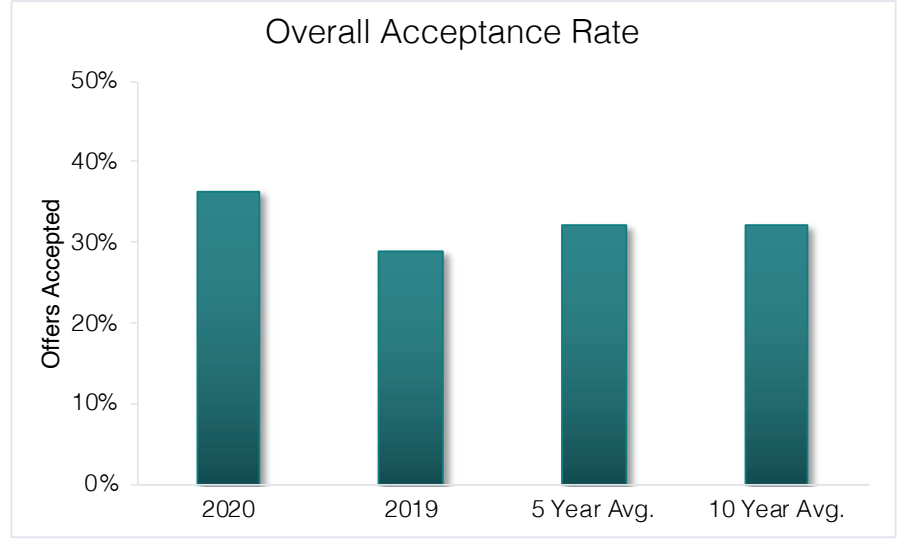

Figure 20. The overall 2020 acceptance rate compared to that of 2019 , the previous five year average, and the previous ten year average.

\section{First Weekend Versus Second Weekend}

Similar to previous years, there was no statistical difference between acceptance rate of the first weekend visitors and the second weekend visitors: $p=0.476$ (2020), $p=0.219$ (2019), $p=0.764$ (five year average), $\mathrm{p}=0.271$ (ten year average) (Figure 21). 


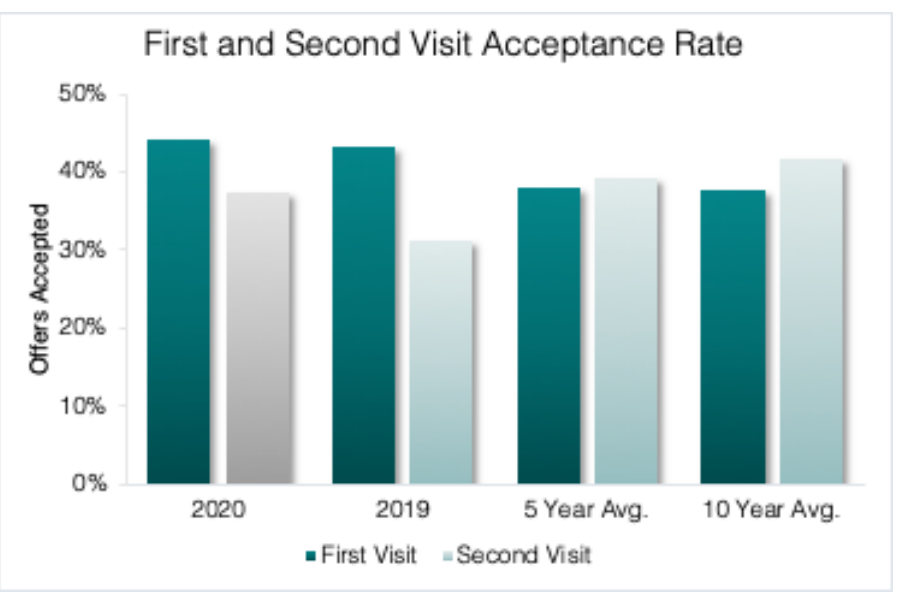

Figure 21. The acceptance rates of students who attended the first and virtual visit of 2020 compared to each other; and compared to the first and second visit acceptance rates of 2019, the previous five year average, and the previous ten year average.

In addition, the acceptance rate of students originally planning to attend the second in-person recruitment weekend that were invited to the virtual recruitment weekend (37.5\%) was not statistically different from the second recruitment weekend acceptance rate of the past year $(31.4 \% \mathrm{p}=0.521)$, the five year average $(39.3 \%, \mathrm{p}=0.816)$, and the ten year average $(41.7 \%, \mathrm{p}=0.577$; Figure 21$)$. This suggests that the Slack virtual recruitment was as effective as the second recruitment weekend of our past.

\section{Non-Visitors}

The acceptance rate of non-visitors (see definition in "Outcomes" section) appeared to be greatly affected by virtual visit attendance. Slack users had a significantly increased acceptance rate (77.8\%) over the previous year $\left(16.4 \%, \mathrm{p}=6.71 \times 10^{-9}\right)$, the five year average $\left(22.4 \%, \mathrm{p}=2.17 \times 10^{-10}\right)$, and the ten year average $\left(24.1 \%, p=2.37 \times 10^{-10}\right)$. The non-Slack user acceptance rate was significantly lower $(2.00 \%)$ than previous years: $p=0.005$ (2019), $p=4.00 \times 10^{-4}$ (five year average), $\mathrm{p}=2.00 \times 10^{-4}$ (ten year average).

The overall acceptance rate of Slack and non-Slack users (28.6\%) was only significantly higher when compared to that of 2019 ( $\mathrm{p}=0.0417)$, while it remained consistent with the acceptance rates of the five year average $(p=0.252)$ and the ten year average $(p=0.385)$ (Figure 22). 


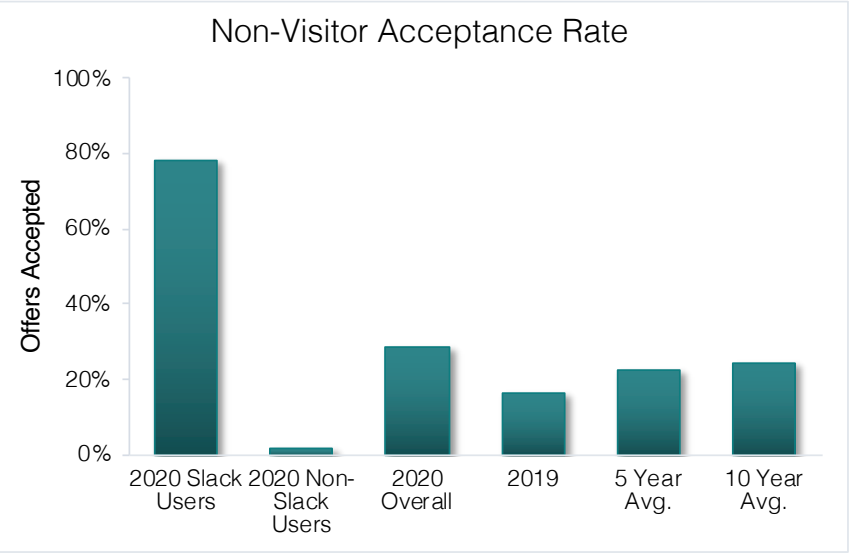

Figure 22. The acceptance rate of non-visiting students in 2020 compared to the non-visiting acceptance rate of 2019, the previous five year average, and the previous ten year average. The acceptance rates of non-visiting Slack users and non-visiting non-Slack users are also shown.

\section{Slack Users Versus Non-Slack Users Both Weekends}

The collected data was then divided by global attendance of the Slack virtual recruitment--whether or not a student registered for Slack and viewed content. Slack visitors had a significantly higher acceptance rate $(58.3 \%)$ than the non-Slack visitors $\left(11.1 \%, \mathrm{p}=5.40 \times 10^{-12}\right)$ (Figure 23).

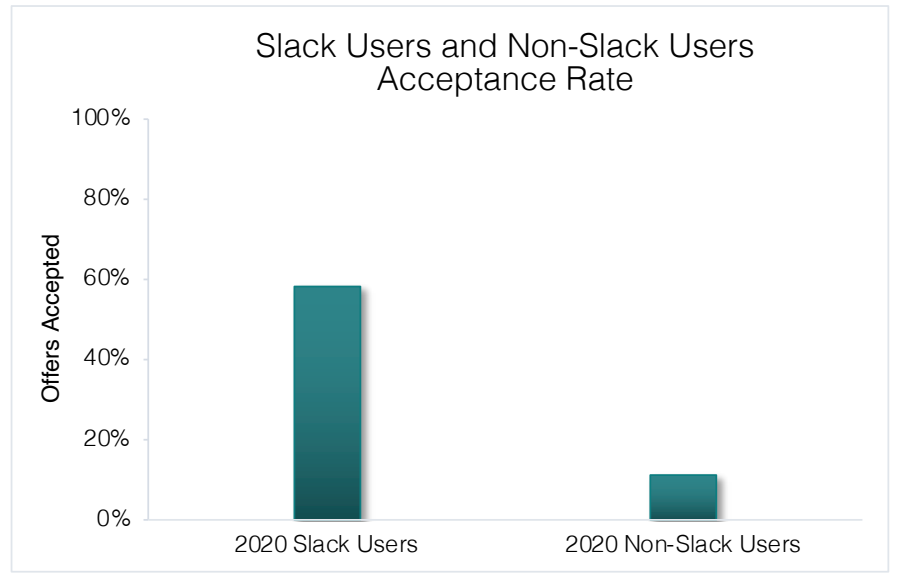

Figure 23. The 2020 acceptance rate of Slack-users compared to that of non-Slack users.

2020 Slack Users Increased Acceptance Rate of First Weekend Visitors 
Figure 24 shows that students who attended both the first in person recruitment weekend and the second Slack virtual recruitment weekend had a significant increase in acceptance rate (65.6\%) than the three comparative data sets $43.1 \% \mathrm{p}=0.0230$ (2019), $37.90 \% \mathrm{p}=0.00147$ (five year average), and $37.8 \% \mathrm{p}=0.00103$ (ten year average).

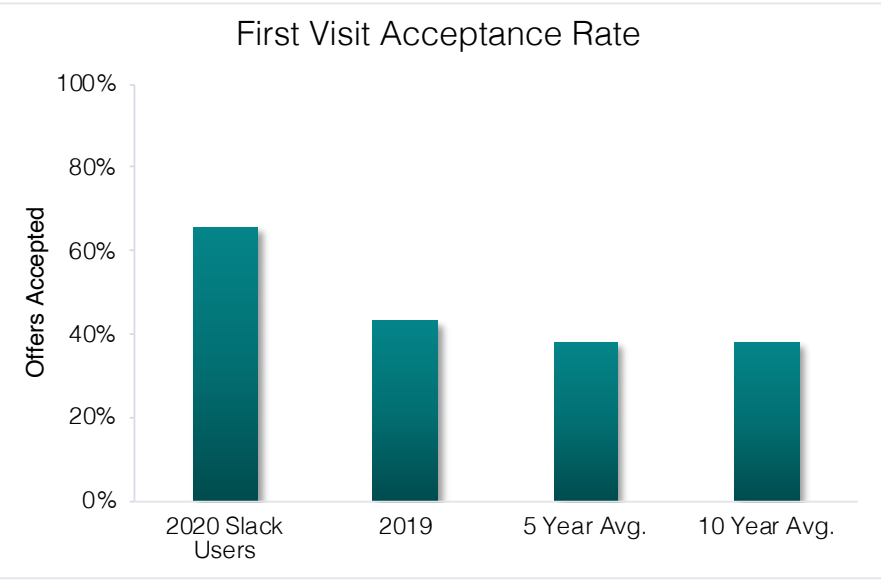

Figure 24. The 2020 acceptance rate of students who attended the first visit and were also present on Slack compared to the first visit acceptance rate of 2019, the previous 5 year average, and the previous ten year average.

Over the course of the spring semester, most universities transitioned to online recruiting at some stage. Although supporting data is not available, one could envision several baseline scenarios for graduate recruiting outcomes. For example, one might hypothesize that prospective graduate students would be more likely to accept an offer from a school they visited in-person. In this scenario, for schools that had one virtual and one in-person weekend (like UMN), the expected outcome would be an increase in acceptances from the in-person weekend with a corresponding decrease in acceptances from the virtual weekend. In an alternate scenario there is no baseline difference in acceptance rate between virtual and in-person weekends.

We saw an increase in acceptances for the first weekend. There was also no statistically significant increase or decline in acceptances from the second virtual recruitment weekend (Figures 20 and 24). Taken together, these observations could lead to a conclusion that digital recruiting via Slack was potentially more successful than other universities' efforts. Our increase in the first acceptance rate 
could be an effect of those students being able to access content via Slack after their in-person visit, or because students were uncomfortable accepting offers from other schools that they were unable to visit in-person. This explanation could also hold true for non-visitors as Slack content, up to date information about research groups, and individual group members could be easily accessed. Overall, students from all categories were more likely to accept an offer to UMN if they had visited the Slack workspace.

In addition to acting as an effective recruitment tool, Slack recruiting achieved several other positive outcomes for our department. First, prospective students could access materials such as posters, presentations, and videos after the weekend. This allowed them to answer questions on their own and to explore groups they did not have time to "meet" with during the virtual recruitment. Quite remarkably, the Slack recruitment channel has maintained an active user base long after the recruiting weekend: for example, incoming students created a Slack channel to introduce themselves and coordinate housing and other details. This type of community-building effort is an excellent example of the positive effect of low-barrier online communication tools. An additional positive outcome was that faculty and students became familiar with virtual communication prior to the UMN work-from-home order that was given the following week. In fact, numerous research groups and instructors in the UMN chemistry department adopted Slack to remain connected to their groups or classes during the shutdown. Faculty and students also received in-person training on how to use Zoom, the primary platform for virtual teaching at UMN, rather than having to scramble at the last minute. Adoption of diverse online tools prior to shutdown was an important anxiety-reducing benefit for many members of our community.

\section{Conclusions and Outlook}

\section{Visitor feedback:}

"I had a great time, which came as a pleasant surprise. I was really looking forward to the actual visit and was disappointed at the circumstances, but this visit still managed to show what I hoped to learn about UMN."

"Y'all did a really good job in a difficult situation." 
"It worked out very well. I felt like I got enough of a feel of the department to make a good decision."

In conclusion, we have shown that an integrated virtual recruitment weekend on Slack can be equally effective to an in-person recruitment weekend. By developing a virtual recruitment weekend platform using Slack, we were able to make graduate recruitment weekend content and interactions with faculty and current students accessible to our prospective students. Looking forward, virtual recruitment weekends are well-positioned to increase diversity, equity, and inclusion around graduate recruiting. They can be used to reach out to students who cannot travel due to illness, monetary constraints, or overlapping in-person weekends. Additionally, virtual recruitment weekends provide an opportunity to increase inclusion of prospective international graduate students, who are often left out of "active" recruiting because of time and money constraints. "I think it is a great experience. Also, I suggest that the Chemistry Departmen [sic] should continue doing this activity for the international students like me, because sometimes is complicated to travel abroad and visit the University in person, so this could be a helpful and powerful tool." In fact, after the virtual visitation weekend plan was announced to our current graduate students, many international students were thrilled with the opportunity to connect with the international prospective student cohort and begin to build community. We also envision this platform can be used for a "pre-recruitment" weekend before applications are due in order to target students from underrepresented minorities, HBCUs, women's colleges, and universities that serve first generation or low income students. This will encourage increased interactions with current students and especially faculty, a commonly-cited factor in decisions by underrepresented minorities. ${ }^{1,2}$ This will increase the diversity of the prospective student pool we are able to reach. We also envision offering a virtual recruitment weekend to students who are interested in decreasing their carbon footprint by eliminating travel.

\section{ASSOCIATED CONTENT}

The Supporting Information is available on the ACS Publications website at DOI:

10.1021/acs.jchemed.XXXXXXX. 


\section{AUTHOR INFORMATION}

Corresponding Author

*E-mail: itonks@umn.edu and ccrob@umn.edu

\section{ACKNOWLEDGMENTS}

We would like to thank the graduate students, postdocs, faculty, support staff, IT staff, student groups, and leadership of the UMN Department of Chemistry for working tirelessly to create content and Slack channels, participate in live events, and adapt so quickly to the change of format. We would like to especially acknowledge

David Blank and Aaron Massari for their contributions to the implementation of the virtual recruitment weekend.

\section{REFERENCES}

(1) Rogers, M. R.; Molina, L. E. Exemplary Efforts in Psychology to Recruit and Retain Graduate Students of Color. American Psychologist 2006, 61 (2), 143. https://doi.org/10.1037/0003066X.61.2.143.

(2) Griffin, K. A.; Muñiz, M. M. The Strategies and Struggles of Graduate Diversity Officers in the Recruitment of Doctoral Students of Color. Equity \& Excellence in Education 2011, 44 (1), 57-76. https://doi.org/10.1080/10665684.2011.540961.

(3) Quarterman, J. An Assessment of Barriers and Strategies for Recruitment and Retention of a Diverse Graduate Student Population. College Student Journal 2008, 42 (4), 947-967.

(4) Kim, S. S.; Gelfand, M. J. The Influence of Ethnic Identity on Perceptions of Organizational Recruitment. Journal of Vocational Behavior 2003, 63 (3), 396-416. https://doi.org/10.1016/S0001-8791(02)00043-X.

(5) Golde, C. M. Beginning Graduate School: Explaining First-Year Doctoral Attrition. New Directions for Higher Education 1998, 1998 (101), 55-64. https://doi.org/ 10.1002/he.10105.

(6) harva015. Diversity \& Inclusion Committee https://chem.umn.edu/about-us/diversityinclusion-committee (accessed Jun 16, 2020).

(7) Perkel, J. M. How Scientists Use Slack. Nature News 2017, 541 (7635), 123. https://doi.org/10.1038/541123a.

(8) How Slack-Ing Helps Chemists Manage Their Labs. C\&EN Global Enterp 2016, 94 (29), 23-24. https://doi.org/10.1021/cen-09429-scitech1. 